\title{
Future heat extremes likely to have been underestimated
}

3
N. Freychet ${ }^{1}$, G. Hegerl' ${ }^{1}$, D. Mitchell ${ }^{2}$, M. Collins ${ }^{3}$

${ }^{1}$ School of Geosciences, University of Edinburgh, Edinburgh, UK

${ }^{2}$ School of Geographical Sciences, University of Bristol, Bristol, UK

${ }^{3}$ College of Engineering, Mathematics and Physical Sciences, University of Exeter, Exeter, UK

Corresponding author: nicolas.freychet@ed.ac.uk

In a warming world, temperature extremes are expected to show a distinguishable change over much of the globe ${ }^{1}$ and in many regions this change has already been detected in observations ${ }^{2,3}$. Although previous studies predict an increase in heat extreme events, the magnitude of the change varies greatly among different models even for the same mean warming ${ }^{4}$. This uncertainty has been linked to differences in land-atmosphere feedbacks across models ${ }^{2}$. Here we show that a significant constraint for future projections can be based on the ability of climate models to accurately simulate the variability of daily atmospheric surface maximum temperature (TX). By applying an emergent constraint (EC) locally on a 
metric describing TX variability with a large ensemble of CMIP5 ${ }^{5}$ and CMIP6 ${ }^{6}$ models we demonstrate that the best estimate increase in hot extremes could be worse than previously estimated over a large part of the land, with an increase in extremes of up to $\mathbf{5 0 \%}$ larger than based on the multi-model mean. Our findings highlight the importance to correctly simulate TX variability during the historical period. Analysis of models soil moisture suggests that the EC arises because both TX variability and changes in hot extremes are related to land surface humidity processes.

Temperature extremes impact strongly on society and can have negative consequences on health ${ }^{7}$, agriculture ${ }^{8}$ or water resources ${ }^{9}$. Daily maximum temperature (TX) is often used to measure heat wave intensity. It is governed by many processes, including accumulation of solar radiation, heat transport, and sensible and latent heat flux exchange with the surface. Particularly, energy used to evaporate surface moisture can limit atmospheric warming and thus $\mathrm{TX}^{10}$. At any given location $\mathrm{TX}$ tends to be larger under drier surface conditions than wetter conditions. Another way to formulate this idea is that soil moisture (and other surface humidity variables) deficit can lead to amplified TX (and with it, potentially amplified heat waves). There is evidence that many current climate models dry too much ${ }^{11}$ and we hypothesize that this amplifies TX variability (thus heat wave frequency ${ }^{12}$ ) whereas more accurate models may see this amplification in the upcoming decades. We postulate that this could lead to large differences between models in terms of heat wave changes under climate warming. 
Many indices of TX can be used to describe hot events (as defined by Expert Team on Climate Change Detection and Indices, ETCCDI). We chose a simple derived index that can be applied easily at global scale, namely the number of days above the $98^{\text {th }}$ percentile (TX98p, see methods for detailed computation). We only focus on the warmest season (June to August for North Hemisphere, December to February for South Hemisphere and all year for the $15^{\circ} \mathrm{S}-15^{\circ} \mathrm{N}$ tropical area). TX98p indicates for each location when a day is considered as extremely hot (relative to the daily climatology of TX at this location). By definition, it represents the $2 \%$ hottest days during the baseline period (1995-2005) at each location, and we evaluate its change in climate projections (see methods for details). We also define a metric to quantify the historical variability of $\mathrm{TX}$ at each location, $\Delta \mathrm{TX}$. This metric indicates at each grid point and for each calendar day the distance between mean TX and the $95^{\text {th }}$ percentile of TX (TX95p) in degrees C. $\Delta T X$ gives an indication of the temperature difference between a hot day compared to the climatology. It is used to evaluate models against a reference dataset, ERA5 reanalysis ${ }^{13}$. This difference has been found to be too high in some climate models (e.g. 14). Computation of $\Delta T X$ implies that we ignore any bias in the mean TX of a model (compared to ERA5) and focus only on TX variability. Note that our results are not sensitive to using another threshold for heat wave index, e.g. the $95^{\text {th }}$ percentile instead of $98^{\text {th }}$ percentile (displayed in supplementary information).

Previous studies have shown that soil moisture deficits enhance surface temperature extremes ${ }^{15,16}$ and have a strong impact on severe events such as heat waves ${ }^{17}$. Here we focus on daily timescale temperatures and due to limited availability of humidity model 
67 outputs at high temporal resolution (especially evaporation and integrated soil moisture

68 are not available at daily timescales for CMIP5 outputs) we use the upper layer of soil

69 moisture (called USM in model data, here referred to as Upper layer Soil Moisture USM

70 for simplicity) as an indicator of land-atmosphere humidity interaction. Although USM is

71 controlled by several factors such as infiltration, horizontal transport and evaporation

72 (with parametrisation varying with land surface models), we assume it can be an

73 indicator of land surface conditions during hot days. We verified that USM conditions

74 during days above the $98^{\text {th }}$ percentile exhibit a negative correlation with $\Delta T$ TX over $80 \%$

75 of the land (Fig.S1,a), i.e. models with highest $\Delta T X$ are also drying the most. This

76 confirms the relationship between surface humidity and TX variability during the

77 baseline period. For some regions this relationship is not or poorly verified. This may be

78 due to other variables influencing humidity and not included in our analysis (e.g.

79 vegetation, deeper layer soil moisture or irrigation), specific land properties (such as

80 permafrost for northern regions) or simply because the number of individual models for

81 this analysis is limited. Thus even if we consider hereafter $\Delta T X$ as an indicator of model

82 historical performances in surface humidity feedback, the physics of the relationship

83 could be closer explored in each model. 

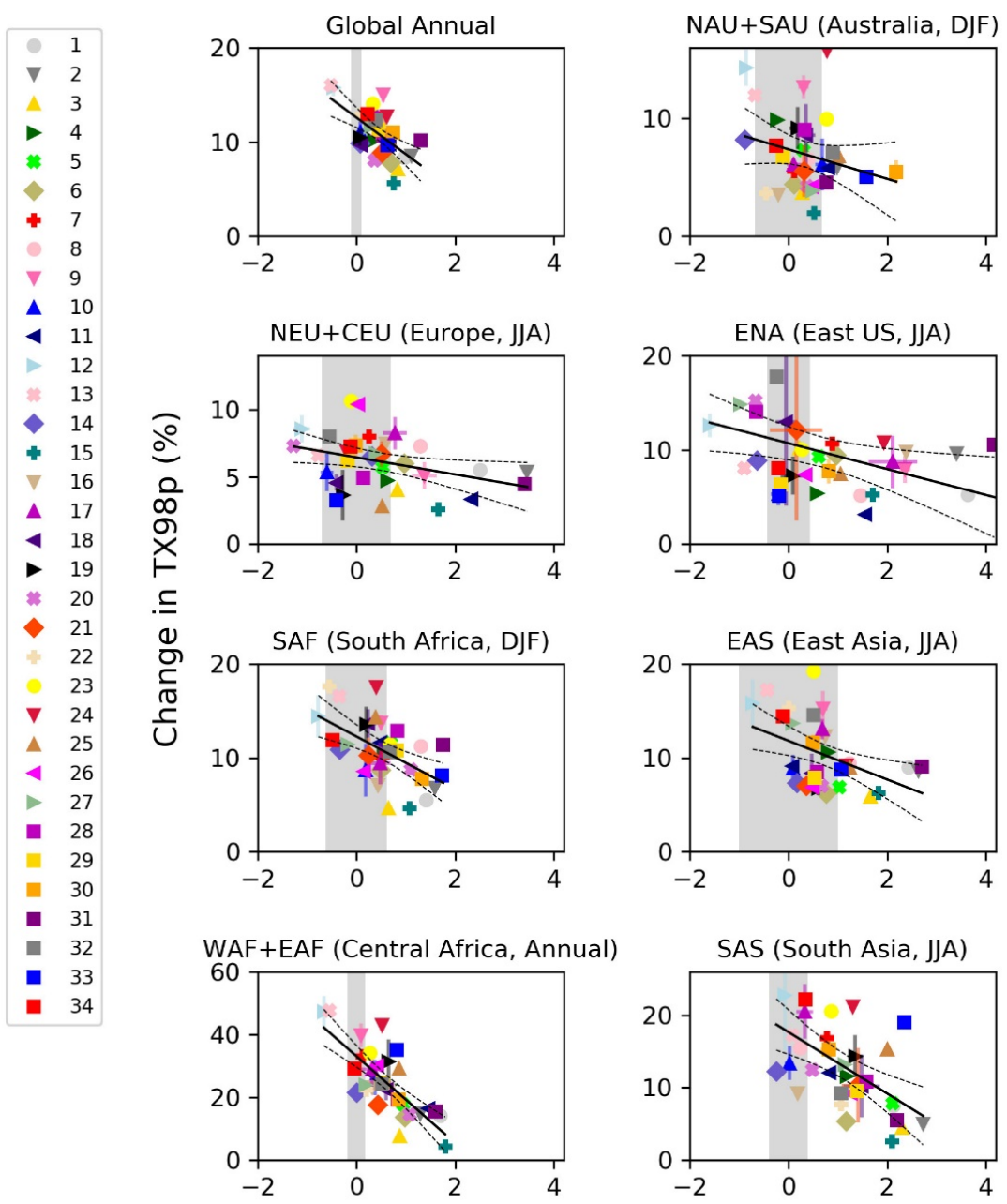

\section{Fig.1: Relationship between $\Delta \mathrm{TX}$ and projected change in TX98p in selected regions}

87 The figure shows for each CMIP5 and CMIP6 models the change in the ensemble average frequency of

88 hot days (TX98p, y-axis, in \% of days) in the future (last decade of rcp45 and ssp245) compared to the

89 present period (1995-2005) plotted against the a variability metric for daily maximum temperature $(\Delta T X)$

90 during the historical period (x-axis, in ${ }^{\circ} \mathrm{C}$ ) averaged over different sub-regions. $\Delta \mathrm{TX}$ measures the

91 difference between daily TX95p and mean TX in a model compared to that observed. Solid black line is

92 the linear regression between $\Delta T X$ and TX98p, and dashed black lines show the $95 \%$ confidence interval.

93 Grey shading represents $\triangle T X$ uncertainties estimated from HAPPI ensemble. Acronyms refer to AR5

94 region definitions and numbers refer to models in Table 1. 
96 We also verify that $\Delta \mathrm{TX}$ is strongly correlated to TX98p change for different warming

97 targets. Over most of land the relationship between $\Delta T X$ and TX98p change is negative

98 (Fig.1) and significant (Fig.S2), indicating that in regions with overestimated variance for

99 hot days the future change in TX98p is smaller on average. Thus, this simple metric is

100 justified to constrain model projections. In the following we mask results only where

$101 \Delta$ TX-TX98p correlation is significant. It is the case at global scale (figure 1), where the

102 metric indicates a tendency to too large $\Delta T X$ for most models, and over most of regions

103 except central North America, central Europe and northern polar regions.

105 The EC methodology requires understanding and accounting for observational and 106 model variability and uncertainties so they it can decide how consistent they $\operatorname{are}^{18}$. We

107 use the internal variability of a large multi-member historical ensembles (HAPPI) that 108 was forced with observed sea surface temperatures to estimate $\Delta T X$ variability at each

109 location. We then consider this information as an uncertainty range for $\Delta T X$ based on

110 ERA5 and to evaluate when models fit within this range (with multi-member models

111 having narrower uncertainty, see methods). 
(a) All models

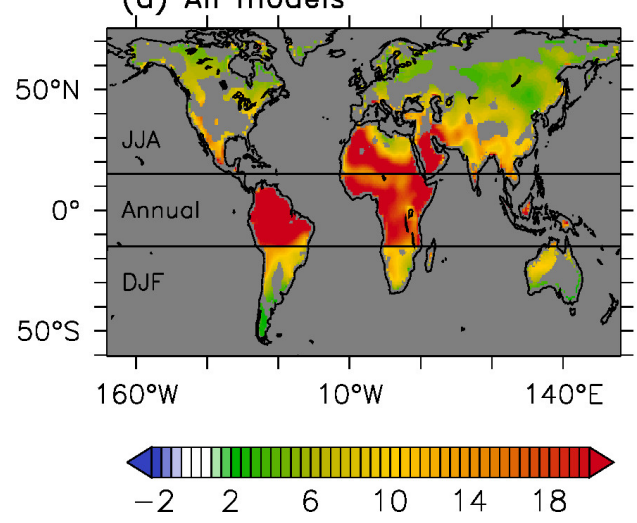

(b) Best models vs All models

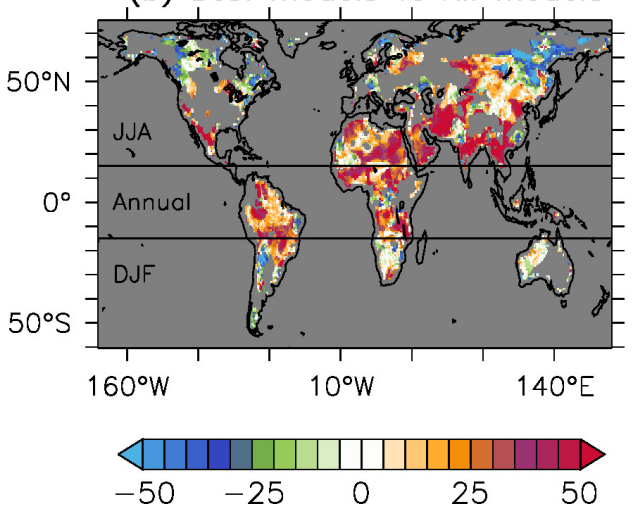

(c)

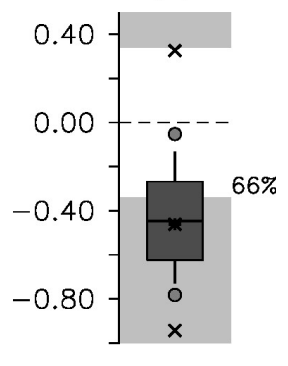

Fig2: Implication of emergent constraint for future change in extremes

115

(a) Ensemble mean (all CMIP models) difference in TX98p per degree warming compared to the baseline 1995-2005 period, expressed as a percentage of days (+X\% means an extra X\% of days each year will be above the $98^{\text {th }}$ percentile, see methods). (b) Difference in TX98p projections between models that reproduce the observed constraint and all models, expressed as a percentage of the change in (a). (a) and (b) display results only where the correlation between TX98p and $\Delta T X$ is significant (see supplementary Fig.S2). (c) Box plot distribution of cross-models correlation coefficients between historical $\Delta T X$ and change in TX98p computed at each grid point. Dark grey box is the $25-75$ interquartile, with horizontal bar inside being the median; vertical solid black line shows the 10-90 interquartile; lower and upper circle symbols are percentile 5 and 95 respectively; lower and upper cross symbols are percentile 1 and 99 respectively. Black cross in the box is the mean. Only values in light grey shading are significant at the $95 \%$ confidence level. The percentage written indicate how many grid points are above this confidence level.

Using $\Delta T X$ to constrain climate projections by selecting in each gridbox (after spatial smoothing, see methods) the models within the observed constraint, we found that changes in TX98p are larger than estimated by an unconstrained ensemble over a large 
131 part of the land (Fig.2). Africa, South and Central Asia and South America have a 132 particularly strong signal, locally above $50 \%$ increase in the number of exceedances of

133 the $98^{\text {th }}$ percentile (although the magnitude of the difference may be partly explained by 134 the number of selected models at each location); i.e. twice as many hot days as in 135 unconstrained predictions. This means models representing more accurately $\Delta T X$ 136 during baseline period (and hypothetically humidity feedbacks) tend to warm faster 137 compared to the other models. Similar relationships are found for all climate warming 138 targets (supplementary Fig.S11 and S12), although the area with significant correlation 139 is reduced for $1.5^{\circ} \mathrm{C}$ target. This influence of our EC persists through different warming 140 targets and is confirmed robust by several sensitivity tests (see methods).

141 Applying an EC based on global mean $\triangle T X$ (i.e. selecting or rejecting a single model on 142 a global mean relationship) leads to slightly weaker, but still valid, amplification (Fig.3). 143 Using a regional constraint to select the best models at each location seems more 144 appropriate, as no model is considered good everywhere (supplementary Fig.S3). The 145 constrained TX98p signal (either by local or global method) suggest that the level of 146 increase previously estimated by the end of the century could be reached by 2060 147 instead, i.e. 40 years earlier. All these results are verified independent of model 148 selection by performing sensitivity tests where one model is removed randomly from the ensemble (Fig.3). The regional constraints results remain highly consistent. The global 150 constraint is still consistent but slightly more sensitive to model selection (due to the 151 small size of this ensemble that fall near the uncertainty range). Thus using regional 152 constrain method here leads to more stable and reliable results. 


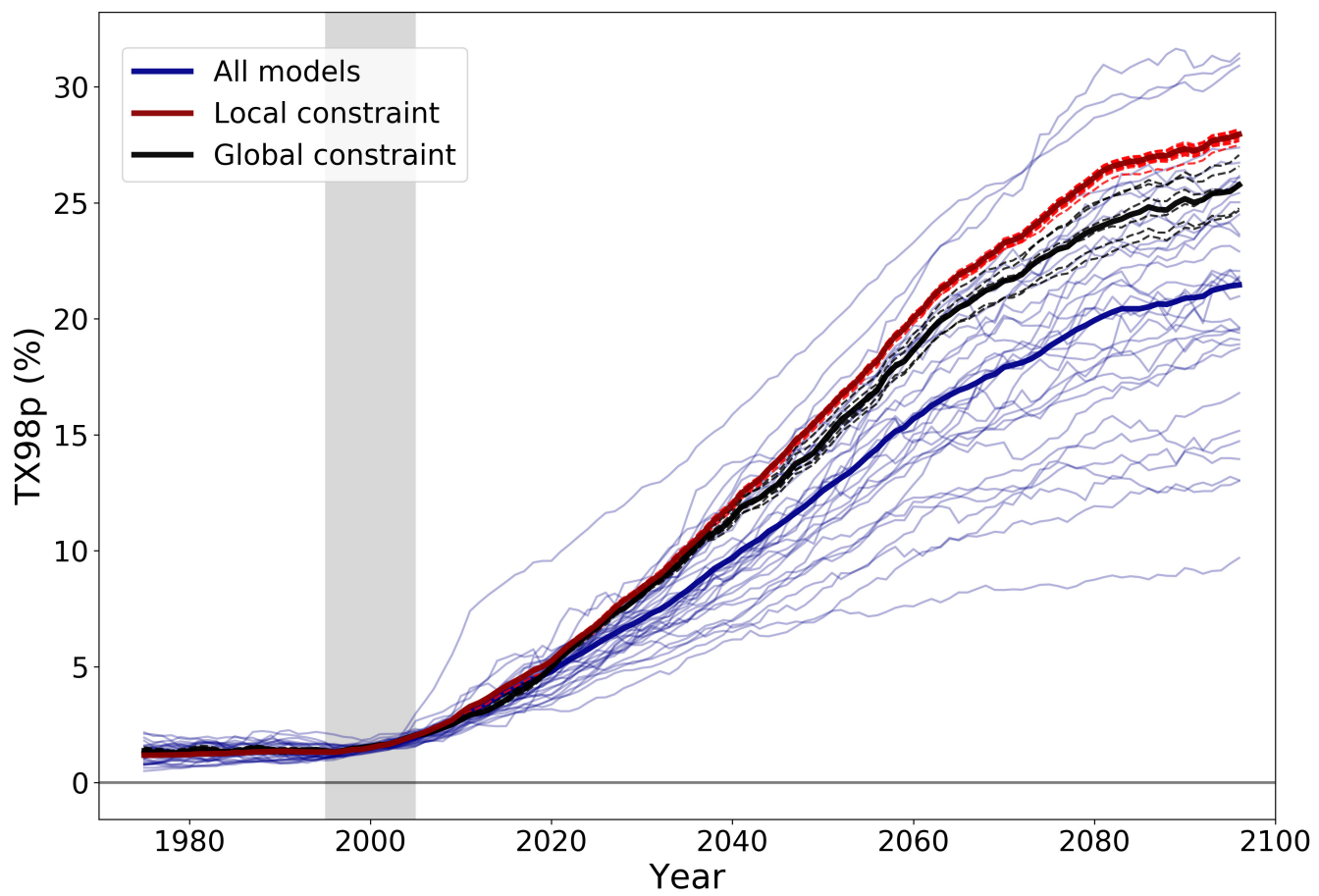

Fig.3: Global evolution of hot extremes in unconstrained and constrained ensembles.

Timeseries of global mean TX98p (\%) for the mean (thick solid line) of all CMIP5 and CMIP6 models

(blue) and constrained models with constraint applied to each significant grid point (red). Ensemble

means are computed, each year, for each grid point from a 9-years running mean of TX98p, then globally

averaged to obtain a global mean value. This method allows a more detailed computation of constrained

ensemble, as the number of models varies from one region to another. Thin blue lines indicate individual

model results. Solid back line shows the mean of a sub-ensemble (7 models) where EC is based on

globally averaged $\Delta T X$ (instead of applying EC at each grid point). Gray shading highlights the baseline

172 each model of each ensemble). For each model, TX98p is linearly scaled by comparing its individual

173 change in Tas to the ensemble mean change in Tas. 
177 mean land drying, not specifically during hot days). The relationship between change in

178 TX98p and mean USM is overall negative, indicating larger temperature variability for

179 drier soils, and supporting our hypothesis (supplement material Fig.S1,b), although it is

180 significant only over few areas and not necessarily where EC signal is the strongest

181 (especially, it is weak over the tropical area). This may be explained by several reasons.

182 First, USM is only one part of land moisture and does not include vegetation (which can 183 be an important factor moderating humidity over tropical land). Secondly due the limit of 184 USM model output data to monthly we may not capture the specific heat event well 185 enough, making the statistical relationship more difficult to estimate. Third, a full daily 186 analysis on evaporation, vegetation and soil moisture structure would be needed to 187 understand how these processes changes under very specific conditions (hot days). 188 This is obviously a strong limit to our current understanding and we can only raise a 189 physical hypothesis. We stress here the importance of high temporal resolution surface 190 humidity outputs to fully understand extreme event processes and humidity feedbacks.

191 We note that over some regions constrained models do not indicate an increase in 192 TX98p, especially over northern part of America and Siberia. These correspond to areas 193 with weak correlation between $\Delta T X$ and TX98p. Other processes may be more 194 dominant in these regions, and drying of soil may be not a factor in high latitudes. 195 Additionally, permafrost land-atmosphere exchanges and humidity processes are 196 different there.

197 Overall, our results indicate that climatological bias in the difference between hot and 198 average days in climate models lay lead to an underestimate of the frequency of 199 unusually hot days in the future. 


\section{Acknowledgment}

203 We acknowledge the E-OBS dataset from the EU-FP6 project UERRA

204 (http://www.uerra.eu) and the Copernicus Climate Change Service, and the data

205 providers in the ECA\&D project (https://www.ecad.eu). This research used science

206 gateway resources of the National Energy Research Scientific Computing Center, a

207 DOE Office of Science User Facility supported by the Office of Science of the U.S.

208 Department of Energy under Contract No.DE-AC02-05CH11231. This research is

209 funded by NERC grant award NE/S004661/1 EMERGENCE project. 


\section{METHOD}

216

217

218

219

220

221

222

223

224

225

226

227

\section{Definition and computation of indices}

Our analysis focusses on daily maximum temperature (TX) extremes (TX98p). We define TX98p as the number of days above the daily climatological $98^{\text {th }}$ percentile. The latter is computed for each location and each calendar day by pulling together all days within +/- 15 days window of this calendar day during the 1995-2005 period and selecting the $2 \%$ highest values.

We also define a metric, $\Delta T X$, to evaluate the variability of TX during the baseline period. It is done by first calculating the mean and $95^{\text {th }}$ percentile of the temperature distribution for each calendar day at each location (by pulling 15 days around each calender day together as for $98^{\text {th }}$ percentile describe above). The distance between the $95^{\text {th }}$ percentile and the mean gives an indication of TX variability for each day and each location. It is computed for each model $(\Delta \mathrm{TX}$ model $)$ and for the reference dataset $\left(\Delta \mathrm{TX}_{\text {ref; }}\right.$; the ERA5 reanalysis) and the difference between the two defines our metric: $\Delta T X=$ $\Delta \mathrm{TX}_{\text {model }}-\Delta \mathrm{TX}_{\text {ref. }}$ We only focus on the warm season, when hot extreme are likely to happen (June-August for North Hemisphere, December-February for South Hemisphere and all year for the $15^{\circ} \mathrm{S}-15^{\circ} \mathrm{N}$ tropical area). Positive values mean a model overestimates the TX variability compared to the reference (i.e. it tends to warm up too quickly and over-estimated high values of TX), negative values indicate an underestimate. For the metric, we choose the $95^{\text {th }}$ percentile to ensure reasonably good sampling of the variability across the base period (as it is used to constrain models) while for future changes we focus on the $98^{\text {th }}$ percentile which correspond to more extreme values. We verified that EC results are not very sensitive to the choice of 
238 threshold by doing a sensitivity test using the $95^{\text {th }}$ percentile as threshold instead $98^{\text {th }}$

239 (supplementary Fig.S4).

240 Each index is computed individually for each model (and eventually each member) on

241 their native grid. Results are then interpolated on a common $1^{\circ}$ grid before being

242 averaged across all models. As temperature extremes are relatively large-scale, and

243 grids vary only between 1 and 2.5 degrees latitude/longitude across models, results are

244 not sensitive to the order of operation.

\section{Datasets}

- CMIP models

248 An ensemble of 27 individual models from CMIP5 5 and 7 from CMIP6 $^{6}$ is used. Some 249 only have a single member available while some provide a multi-members ensemble. In 250 the latter case, multi-member results are always computed individually and then

251 averaged to provide one mean result for a single model. We consider a reference period

252 as the historical 1995-2005 decade (being the last decade of CMIP5 historical forcing).

253 Climate projections are investigated using the RCP $4.5^{19}$ and SSP $245^{20}$ pathways for

254 CMIP5 and CMIP6 models respectively. Both scenarios are expected to be close,

255 although each model leads to different mean temperature increases (Fig.S5).

256 Three climate projection targets are considered:

257 - end-of-century, by selecting the 2091-2100 decade for each model.

$258-+1.5^{\circ} \mathrm{C}$ and $+2^{\circ} \mathrm{C}$ warming above pre-industrial mean. For these two, we follow a 
259 similar approach as in 21 and select for each member of each model the first decade 260 when the average atmospheric surface temperature (Tas) of each year of the decade is

261 above the corresponding threshold (Fig.S5). As we use 1995-2005 as a baseline, the 262 actual threshold (relative to the baseline) is chosen as $+0.7^{\circ} \mathrm{C}$ and $+1.2^{\circ} \mathrm{C}$ for targets

$263+1.5^{\circ} \mathrm{C}$ and $+2^{\circ} \mathrm{C}$ above pre-industrial respectively, as in the HAPPI experiment

264 design ${ }^{22}$. Although the exact definition of these levels can be sensitive ${ }^{23}$, for this work 265 the main point is that each model or member should reach a similar magnitude of 266 warming. A few members and models do not meet the condition for the $+2^{\circ} \mathrm{C}$ target 267 before the end of the century. For these cases, we select instead the last projection decade 2091-2100. If the mean increase in Tas over this decade is above the threshold $269\left(+1.2^{\circ} \mathrm{C}\right)$ then we keep the model or member. Otherwise we do not include it in the analysis for this projection target. This leads us to discard 4 members.

271 For each climate projection target, results of each member or model are normalised by

272 their respective mean change over the decade (relative to our baseline) in Tas (and then 273 averaged to provide ensemble mean results). Thus, no matter the target projection all 274 results are shown for $+1 \mathrm{C}$ warming above the baseline. We tested the sensibility of the 275 results by using raw results (without normalisation) for each model but both methods 276 lead to very close results in terms of EC amplification (Fig.3 and Fig.S6), although raw

277 results have larger uncertainties. Thus, we largely focus on normalised results in the 278 body of the paper.

279 For most of the models we could get daily TX data for both historical and projection 280 periods. Daily soil moisture data are more limited (9 CMIP5 and 5 CMIP6 models).

281 Supplementary Table 1 provides details about outputs used for each variable. 
284 To evaluate the uncertainties on $\Delta T X$ during the baseline period we use several 285 atmospheric models from the HAPPI ensemble ${ }^{22}$. Each model provides daily output for 286 the 1995-2005 decade. We select 5 models with a hundred or more members and 287 compute $\Delta \mathrm{TX}$ for each member (same method as for CMIP models). Then, using 288 internal variability of each model (multi-members ensemble standard deviation, $\sigma$ ), we 289 estimate $\Delta T X$ uncertainties for each location and calendar day (Fig.S7). One model has a mean bias that is much larger than other models (CanAM4), we thus exclude it. For other models, the $\Delta T X$ internal variability is consistent, so we use the mean of four remaining model variabilities (i.e. averaging the four internal STD) as a measure of $\Delta T X$ 293 uncertainties $\left(\sigma_{\text {HAPPI }}\right)$.

294 The sensitivity of this choice is also tested by using individual model STD instead of 295 ensemble mean (Fig.S8). It shows that results stay consistent for each case. We note 296 that the uncertainty so described is that of atmospheric variability only. However, both 297 the HAPPI ensemble and the ERA5 reanalysis are driven by the same SSTs hence this 298 choice is conservative to characterize observational uncertainty.

299 Internal variability in the climate models used is reduced by ensemble averaging. To 300 take into account the specific number of members for each individual model, the 301 uncertainty between OBS and models is expressed as: $\left(\sigma^{2}{ }_{\text {HAPPI }}+\left(\sigma^{2}{ }_{\text {HAPPI }} / N\right)\right)^{1 / 2}$ with $N$ 302 the number of members of a model. When the absolute value of $\Delta T X$ fits within that 
303 range then a model (eventually the multi-members ensemble mean) is considered as

304 consistent with OBS.

305

306

$\underline{-E R A 5}$

307 The ERA5 reanalysis ${ }^{13}$ is available for the full satellite observation period (1979-

308 present). It provides hourly timescales data at $0.25^{\circ}$ resolution on a reduced Gaussian

309 grid, from which we computed daily TX for the 1995-2005 period.

310 We evaluated the variability of TX in ERA5 against two dense regional observational

311 datasets (Fig.S9): A network of 756 homogenised station measurements for China,

312 provided by the Chinese Meteorological Administration ${ }^{24}$; And gridded $0.25^{\circ} \mathrm{E}-\mathrm{OBS}$

313 v19.0 dataset for Europe ${ }^{25}$. Chinese observations are first gridded on the same regular

314 grid as ERA5 by linear interpolation.

315 Although the TX variability tends to be weaker in ERA5 than in observations, differences

316 are within the range of uncertainties estimated from the HAPPI ensemble variability

317 (Fig.S7) for both regions, hence we consider ERA5 sufficient.

\section{Emergent constraint (EC) method}

320 To decrease model projection uncertainties on TX98p, we use an EC method with $\Delta T X$ as a predictor (i.e. selecting models that are able to reproduce the width of the daily

322 maximum temperature distribution TX, indicated by the distance between the $95^{\text {th }}$ percentile and the median) and select those for prediction. To do this, CMIP models are 
evaluated against ERA5 during the 1995-2005 period, and agree with it within atmospheric internal variability. We use variability from the HAPPI ensemble to characterize this uncertainty for better sampling. Models (ensemble mean in case of multi-members model) within the range of 2 times STD (i.e. the $95 \%$ confidence interval) are considered as reasonably realistic and selected for use in the constrained climate projections. Comparing constrained against unconstrained ensemble projections provides an estimate of the potential current bias in climate forecasts.

Constraints can arise from global or regional processes ${ }^{18}$. Here we use a regional constraint to take advantage of model information everywhere. We first apply a spatial smoothing of 5 degrees on $\Delta T X$ over land (to improve sampling and avoid spatial discontinuity) then select the models that comply with the constraint within uncertainty at each grid point. Over most of the regions, the number of selected models is between 5 and 10, except in central Africa where it is below 5 . This is mainly due to very narrow observational variability over this region (Fig.S3 and S7). Most of models contribute to the projection over some part of land. Applying EC at a global scale instead (Fig.3 and S10) leads to similar patterns with slightly weaker amplification.

We also tested the sensitivity of EC results with different choices of uncertainty around the observational distribution with and different spatial smoothing (Fig.S8). Using narrower (wider) range of variability leads to slightly different results with less (more) models selected, corresponding to a noisier but more intense (smoother but less intense) signal. However, global patterns are still consistent with main results. Weaker spatial smoothing (3 degrees) leads to slightly nosier results while using too large smoothing (11 degrees) leads to large masked area (because we use only land grid 
347 points or alternatively to large variation in actual applied smoothing). Thus 5 degrees

348 smoothing is a good compromise.

349 Following recommendations from Hall et al., 2019, we first confirm the strong statistical

350 relationship between $\Delta \mathrm{TX}$ and TX98p (Fig.1 and supplement S2). We then use a

351 resampling method (by removing randomly a model from the ensemble) to test the

352 robustness of the constraint (Fig.3). Finally, the physical mechanism hypothesis linking

353 soil moisture, $\Delta T X$ and TX98p is evaluated (Fig.S1), although this evaluation is

354 somewhat limited due to limited soil moisture availability.

Data availability

The authors declare that all data that support the findings in the main article are

359 available. All model data are publicly accessible via the Earth System Grid Federation node

360 (https://esgf-node.ipsl.upmc.fr/). ERA5 data can be downloaded from ECMWF website

361 (https://www.ecmwf.int/en/forecasts/datasets/reanalysis-datasets/era5). Scripts used to generate the

362 main results will be made available on the University of Edinburgh datashare. All other data and code

363 that support the figures in the Supplementary Information are available from the corresponding author 364 on request. 


\section{References}

1: Herring, S. C., Hoerling, M. P., Kossin, J. P., Peterson, T. C., \& Stott, P. A., Explaining extreme events of 2014 from a climate perspective. Bulletin of the American Meteorological Society, 96(12), S1-S172, 2015

2: Seneviratne, S. I., et al., Changes in climate extremes and their impacts on the natural physical environment.. Managing the risks of extreme events and disasters to advance climate change adaptation: Special report of the Intergovernmental Panel on Climate Change, 109-230, 2012

3: Bindoff, N.L., Stott, P.A., AchutaRao, K.M., Allen, M.R., Gillett, N., Gutzler, D., Hansingo, K., Hegerl, G., Hu, Y., Jain, S. and Mokhov, I.I., CH10: Detection and attribution of climate change: from global to regional, 2013

4: Hoegh-Guldberg and Coauthors, $\mathrm{CH} 3$ : Impacts of $1.5^{\circ} \mathrm{C}$ global warming on natural and human systems, 2018

5: Taylor, K.E., R.J. Stouffer, G.A. Meehl, An Overview of CMIP5 and the experiment design. Bull. Amer. Meteor. Soc., 93, 485-498, 2012

6: Eyring, V., Bony, S., Meehl, G. A., Senior, C. A., Stevens, B., Stouffer, R. J., \& Taylor, K. E., Overview of the Coupled Model Intercomparison Project Phase 6 (CMIP6) experimental design and organization. Geoscientific Model Development (Online), 9, LLNL-JRNL-736881, 2016

7: Guo, Y., A., Gasparrini, B. G. Armstrong and Coauthors, Heat wave and mortality: a multicountry, multicommunity study. Environmental health perspectives, 125(8), 087006, 2017

8: Vogel, E., Donat, M. G., Alexander, L. V., Meinshausen, M., Ray, D. K., Karoly, D., Meinshausen, N. \& Frieler, K., The effects of climate extremes on global agricultural yields. ERL, 14(5), 054010, 2019 
9: Zuo, J., Pullen, S., Palmer, J., et al., Impacts of heat waves and corresponding measures: a review. Journal of Cleaner Production, 1-12, 2015

10: Whan, K., Zscheischler, J., Orth, R., Shongwe, M., Rahimi, M., Asare, E. O., \& Seneviratne, S. I., Impact of soil moisture on extreme maximum temperatures in Europe. Weather and Climate Extremes, 9, 57-67, 2015

11: Milly, P. C. D. and Dunne, K. A., Potential evapotranspiration and continental drying. Nature Clim. Ch., 6, 946-949, 2016

12: Schär, C., P. L. Vidale, D. Lüthi, C. Frei, C. Häberli, M. A. Liniger and C. Appenzeller, The role of increasing temperature variability in European summer heatwaves. Nature, 427, 332-336, 2004

13: Hersbach and Coauthors, Operational global reanalysis: progress, future directions and synergies with NWP. European Centre for Medium Range Weather Forecasts, 2018

14: Hanlon H., Hegerl, G.C., Tett, S.F.B., Smith, D. , Can a decadal forecasting system predict temperature extreme indices?. J. Climate, 26, 3728-3744, 2013

15: Seneviratne, S.I., Corti, T., Davin, E.L., Hirschi, M., Jaeger, E.B., Lehner, I., Teuling, A.J., Investigating soil moisture-climate interactions in a changing climate: a review. Earth-Sci. Rev., 99(3), 125-161, 2010

16: Seneviratne, S. I., Wilhelm, M., Stanelle, T., van den Hurk, B., Hagemann, S., Berg, A., ... \& Claussen, M., Impact of soil moisture-climate feedbacks on CMIP5 projections: First results from the GLACE-CMIP5 experiment. Geophysical Research Letters, 40(19), 5212-5217, 2013

17: Miralles, D. G., Gentine, P., Seneviratne, S. I., \& Teuling, A. J., Land-atmospheric feedbacks during droughts and heatwaves: state of the science and current challenges. Annals of the New York Academy of Sciences, 1436(1), 19, 2019 
18: Hall, A., Cox, P., Huntingford, C., \& Klein, S. , Progressing emergent constraints on future climate change. Nature Climate Change, 2019

19: van Vuuren DP, JA Edmonds, M Kainuma, K Riahi, AM Thomson, K Hibbard, GC Hurtt, T Kram, V Krey, J-F Lamarque, T Masui, M Meinshausen, N Nakicenovic, SJ Smith, and S Rose, The representative concentration pathways: an overview Climatic Change. 109(1-2), 5-31, 2011

20: Gidden, M., Riahi, K., Smith, S., Fujimori, S., Luderer, G., Kriegler, and Coauthors, Global emissions pathways under different socioeconomic scenarios for use in CMIP6: a dataset of harmonized emissions trajectories through the end of the century. Geoscientific Model Development Discussions, 12(4), 14431475, 2019

21: King, A. D., Knutti, R., Uhe, P., Mitchell, D. M., Lewis, S. C., Arblaster, J. M., \& Freychet, N., On the linearity of local and regional temperature changes from $1.5 \mathrm{C}$ to $2 \mathrm{C}$ of global warming. Journal of Climate, 31(18), 7495-7514, 2018

22: Mitchell, Daniel, Krishna AchutaRao, I. Bethke, U. Beyerle, Andy Ciavarella, P. M. Forster, Jan Fuglestvedt et al., Half a degree additional warming, prognosis and projected impacts (HAPPI): background and experimental design. Geoscientific Model Development, 10, 571-583, 2017

23: Schurer, A. P., Cowtan, K., Hawkins, E., Mann, M. E., Scott, V., \& Tett, S. F. B. , Interpretations of the Paris climate target. Nature Geoscience, 11(4), 220, 2018

24: Li Z. and Z.-W. Yan, Homogenized daily mean/maximum/minimum temperature series for China from 1960-2008. Atmospheric and Oceanic Science Letters, 2(4), 237-243, 2009

25: Cornes, R., G. van der Schrier, E.J.M. van den Besselaar, and P.D. Jones, An Ensemble Version of the EOBS Temperature and Precipitation Datasets. J. Geophys. Res. Atmos., 123, 2018 